\title{
Role of MR Apparent Diffusion Coefficient in Characterization of Cystic Renal Masses Using 3T MRI
}

Zahra A Saly ${ }^{\star}$, Abou El Ghar Mohamed, Refaat Medhat and Elasaaed Mohamed

Department of Diagnostic Radiology, Benha University, Benha, Egypt

*Corresponding author: Zahra A Saly, Department of Diagnostic Radiology, Benha University, Benha, Egypt, Tel: +20402382478; E-mail: dr_saly_adel@yahoo.com

Received date: August 31, 2018; Accepted date: October 16, 2018; Published date: October 23, 2018

Copyright: (C2018 Saly ZA, et al. This is an open-access article distributed under the terms of the Creative Commons Attribution License, which permits unrestricted use, distribution, and reproduction in any medium, provided the original author and source are credited.

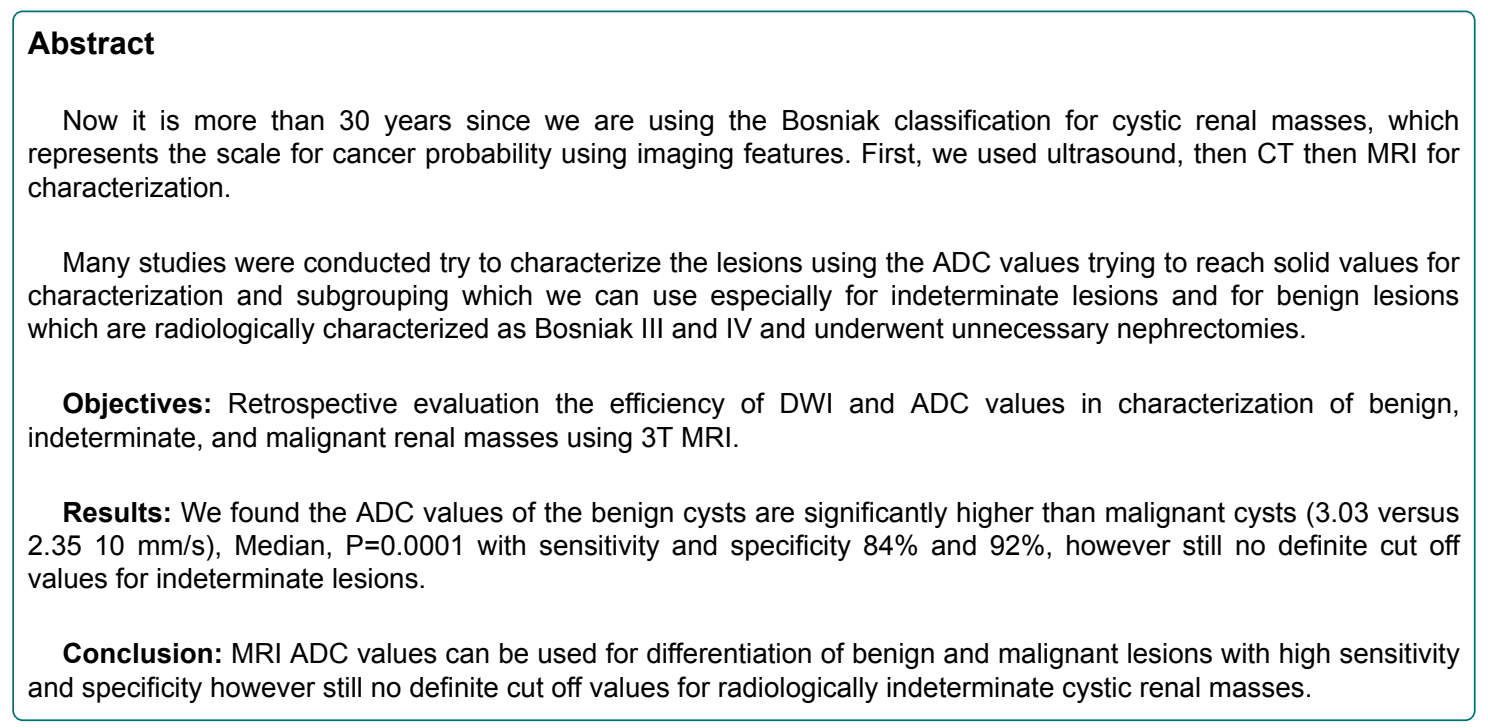

Keywords: Cystic renal masses; Retrospective evaluation; Benign; Malignant lesions

\section{Introduction}

Cystic renal masses are commonly encountered in clinical practice. Improved resolution of imaging modalities has in part led to the improved ability to detect these abnormalities. Yet the Bosniak criteria have stood the test of time as radiologists and urologists still primarily rely on these [1,2].

The conventional CT and MRI sequences cannot easily differentiate benign from malignant lesions in many cases. Studies have shown that $16 \%-33 \%$ of nephrectomies are performed on benign lesions [3].

There is also a strong need for alternatives to gadolinium-enhanced sequences in patients at risk for nephrogenic systemic fibrosis (NSF) [4].

Percutaneous biopsy of renal tumors has been widely demonstrated to be an accurate method of diagnosing preoperative pathologic subtypes in many patients.

However, the risk of procedural complications and the potential for sampling error have hindered universal acceptance of percutaneous biopsy [5-9].

The basic physical principle of diffusion-weighted imaging is based on the random movements of molecules (Brownian motion) in a spatial plane.
This is affected by differences in the nucleocytoplasmic ratio and factors that change water diffusion in the interstitial space such as increased cell density and viscosity [9].

DWI has become more familiar and has gained a definitive role in the characterization of abdominal malignancy in lymph nodes, and renal, liver, pancreas, prostate masses [10].

Diffusion weighted imaging (DWI) has been applied successively in renal masses; however, its usefulness in cystic masses is not established and a well-known pitfall of DWI is that benign HPC may show restricted diffusion [11].

There are still very limited studies using 3T MRI trying for characterization of cystic renal masses using DW-MRI trying to differentiate benign cysts from the malignant cysts which requires surgical interference and indeterminate cysts (IIF).

Our study aims is to retrospectively evaluation of the efficiency of DWI and ADC values in characterization of benign, indeterminate, and malignant renal masses using 3T MRI.

\section{Patients and Methods}

Our retrospective research was approved by Urology and nephrology center board and Benha medical school boards and patient's consents was obtained. 
Citation: Saly ZA, Mohamed AEG, Medhat R, Mohamed E (2018) Role of MR Apparent Diffusion Coefficient in Characterization of Cystic Renal Masses Using 3T MRI. J Nucl Med Radiat Ther 9: 383. doi:10.4172/2155-9619.1000383

Page 2 of 5

\section{Inclusion and exclusion criteria}

We had 73 consecutive patients with 75 cystic renal masses, all the patient underwent MRI scans, malignant lesions underwent biopsy, nephron spring or nephrectomy.

All the scans were obtained prior to biopsy and surgical excision.

In our study we included radiological benign lesions, indeterminate cases which have 2 years follow up, radiologically malignant appearance cases, the scans done before having biopsies or even surgical interference.

The cases with no definitive imaging, clinical diagnosis or no histopathology results were excluded $(n=5)$, patient with no Diffusion MRI sequences were excluded $(n=13)$, lesions with poor quality Diffusion sequences or obvious artefacts are also excluded $(n=4)$.

Our study included finally 51 patients with 51 lesions were included in our study.

\section{MRI Protocol}

All images were obtained using a 3T MR system (Signa horizonGeneral Electric medical systems, Miluakee-USA) equipped with a phased-array coil.

The breath hold DW images were obtained using a customized Black-Blood Spin Echo- Echo Planar Imaging (BB SE-EPI) sequence. An XL TORSO coil was used.

In the coronal T2 TSE Breath Hold (BH) and coronal T2 TSE Free Breathing (FB), two b values (b 800 and b $1600 \mathrm{~s} / \mathrm{mm}^{2}$ ) using the transverse BB SE-EPI sequences were acquired.

\section{The scanning parameters were as follows:}

Coronal T2W TSE BH (TR=shortest, TE=80, Slice Thickness=6, Interslice Gap=1, Matrix $=312$ x 247, FOV FH=405, NSA=1, Sense Factor $=$, Reduction $(R L)=2$, Voxel Size $=0.79$, Slice Number $=74$, Scan Time $=22-3$ ).

Transverse T2W TSE FB (TR=shortest, $\mathrm{TE}=80$, Slice Thickness $=7$, Inter- slice Gap $=1$, Matrix $=284 \times 194$, FOV FH=255, NSA=1, Voxel Size $=0.73$, Slice Number $=32$, Scan Time $=1-36$.

Transverse BB SE-EPI b 800 and b 1600 (Slice Thickness=7, Interslice Gap=1, Matrix $=152 \times 112$, FOV FH=199, RL=450, AP=338, $\mathrm{NSA}=3$, Sense Factor $=$ P, Reduction=2). The DWI acquisition was two minutes and 14 seconds in total.

All of the MR images of the 51 patients were reviewed at the PACS workstation (Philips Workspace, Extended MR Workspace, release 2.6.3. 2009, Philips Medical Systems, The Netherlands).

\section{Image Analysis}

\section{Qualitative assessment including DWI and ADC tumor signals}

We Compared the signal intensity of the lesions in both ADC and DWI images with the normal renal parenchyma in the contralateral kidney or the same kidney if the patient had previous nephrectomy.

We used a rating scale with $1=$ hyper intense, $2=$ hypointense, and $3=$ mixed signal intensity or isointense.

\section{Quantitative assessment using the ADC map}

All the DWI images were transferred for ADC maps reconstruction to an independent workstation for processing. To obtain mean ADCs ( \pm standard deviation) of each cyst we measure ADC maps by using average size circular ADC ROI.

For solid masses with cystic necrosis we placed 2 ROIs on the solid part and on the cystic component. For complex cystic masses we placed the ROIs on either the wall nodularity or the thick septations.

\section{Lymphadenopathy}

Malignant masses with enlarged lymph nodes we assessed the signal intensity and ADC values of the associated enlarged lymph nodes.

\section{Reference standard}

For benign lesions and indeterminate lesions 2 years follow up were obtained to confirm patient stability. For malignant lesions we had either post biopsy or post nephrectomy histopathology results (Figures 1 and 2).

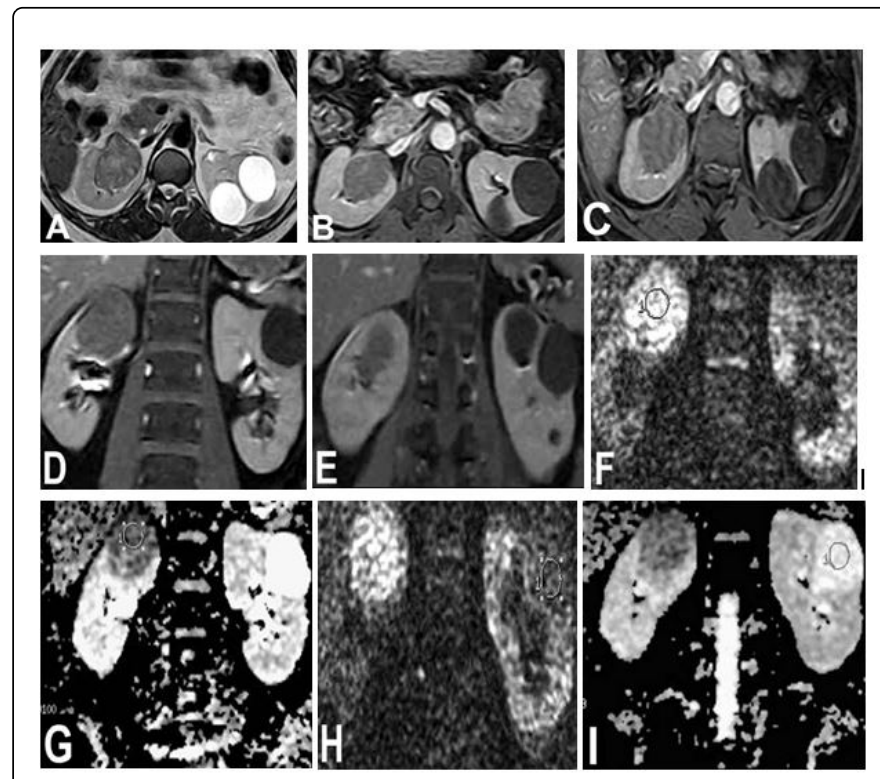

Figure 1: Right RCC (papillary cell type) grade II, and two incidental left simple cysts: A 52-year-old patient with an upper polar right renal mass. (A) Axial T2 FSE: The right renal mass arises off the medial cortex and bulges into the renal sinus. It displays heterogeneous hypo intense T2 signal. The incidental left renal upper polar cysts are T2 bright. (B and C) Axial and (D-F) Coronal post contrast images: The right renal mass shows minimal enhancement, while the left upper polar cysts demonstrate no enhancement. (F and G) DWI and ADC map: The right renal mass shows reduced diffusion with a bright signal on the DWI and hypo intense signal on the ADC map, the mass has a low ADC value due to its malignant nature $\left(0.92 \times 10^{-3} \mathrm{~mm}^{2} / \mathrm{s}\right)$.

\section{Statistical analysis}

Statistical analyses were performed by SPSS version 18.0 (statistical package for social sciences). Data are presented as the mean and 
Citation: Saly ZA, Mohamed AEG, Medhat R, Mohamed E (2018) Role of MR Apparent Diffusion Coefficient in Characterization of Cystic Renal Masses Using 3T MRI. J Nucl Med Radiat Ther 9: 383. doi:10.4172/2155-9619.1000383

Page 3 of 5

standard deviation or $\mathrm{n}(\%)$. The one-sample Kolmogorov-Smirnov test was used to evaluate the distribution of data.

An unpaired Student's t-test was used to compare the mean ADC values between suspicious lesions and normal renal parenchyma after ensuring normal distribution by Shapiro-Wilk test.

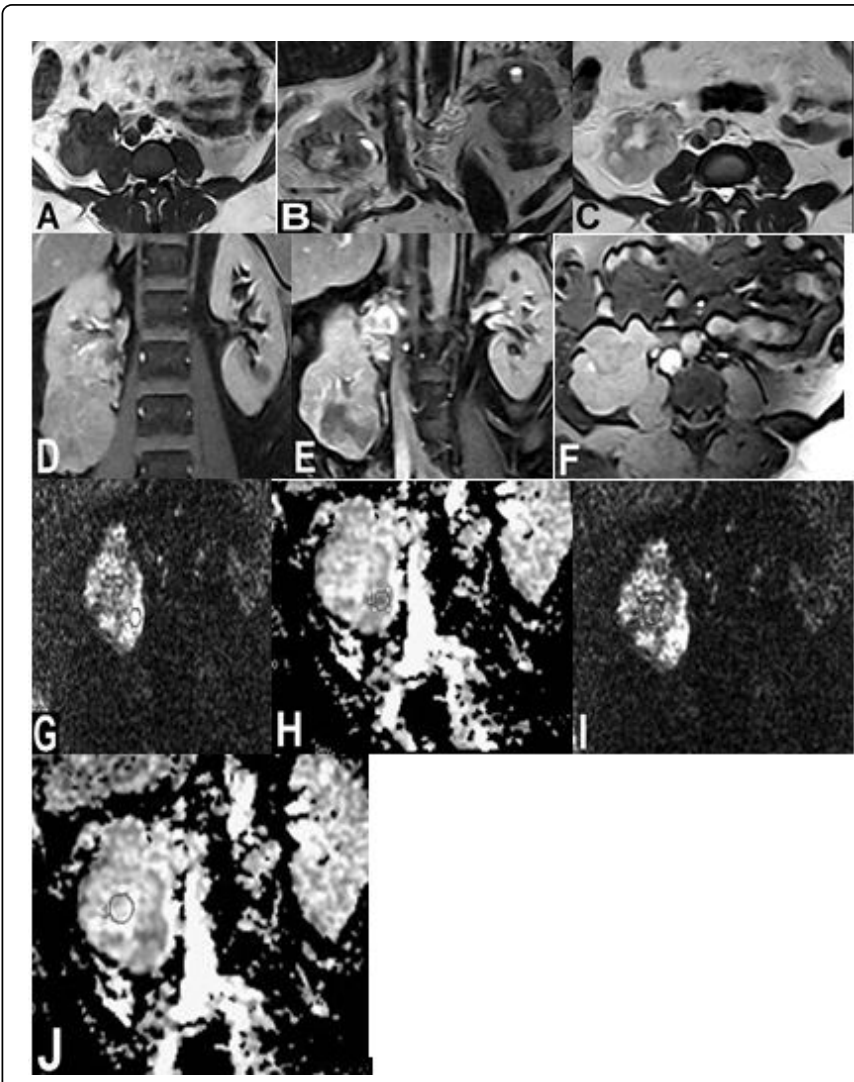

Figure 2: 59-year-old male patient with a complex solid mass in the lower pole of the right kidney with central necrosis.

(A) Axial T1 FSE, (B and C) coronal and axial T2 FSE. (D and E) Coronal and (F) axial post contrast T1 SGRE: The solid part of the mass enhances heterogeneously. ( $G$ and H) DWI and ADC map: The ROI was placed in the solid part which demonstrate restricted diffusion and ADC value of $1.38 \times 10^{-3} \mathrm{~mm}^{2} / \mathrm{s}$. (I and $\mathrm{J}$ ) a second ROI was placed in the cystic necrosis which has ADC value of $2.1 \times$ $10^{-3} \mathrm{~mm}^{2} / \mathrm{s}$ (higher than the solid part but lower than simple cysts).

The Mann-Whitney U or Kruskal-Wallis tests were performed to compare the mean ADCs and histopathological variables.

A p value of less than 0.05 was considered to be a statistically significant difference. Receiver operating characteristic (ROC) analysis was done to evaluate the diagnostic performance of the ADC for differentiating benign and malign lesions.

The significant cut-off value was determined to be the value that best discriminated between benign and malignant in terms of maximum sensitivity and specificity.

\section{Results}

We had 51 patients with 51 renal masses finally, all are enrolled with 31 male patients with 31 lesions and 20 female patients with 20 lesions).

The range of patients age was $24-84$ years, the median age was 63 years (Table 1).

The final diagnosis was confirmed either by stability for 2 years with typical benign radiological features $(n=32)$ or by histopathology examination for malignant lesions.

\begin{tabular}{|c|c|c|c|c|}
\hline & Benign & Malignant & Indeterminate & \multirow{2}{*}{$\begin{array}{l}P \\
\text { Value }\end{array}$} \\
\hline & $n=20$ & $n=28$ & $n=3$ & \\
\hline $\begin{array}{l}\text { Age mean } \\
\text { (SD) }\end{array}$ & $59.8(11.3)$ & $59.5(12.9)$ & $28.0(13.7)$ & $0.001^{*}$ \\
\hline \multicolumn{5}{|l|}{ Sex no\% } \\
\hline 1 & $10(50)$ & $8(28.6)$ & $2(66.7)$ & \multirow{2}{*}{0.1} \\
\hline 2 & $10(50)$ & $20(71.4)$ & $1(33.3)$ & \\
\hline
\end{tabular}

Table 1: Demographic characteristics of studied cases.

\section{Study groups}

We divided the lesions into 3 categories according to radiological features as follows:

\section{- Group 1: Benign cystic renal parenchymal masses}

This group includes 20 lesions.

- Group 2: Indeterminate cystic parenchymal masses

This group includes 3 lesions $(\mathrm{N}=3)$.

\section{- Group 3: Malignant cystic renal masses}

This group includes 28 lesions.

\section{Diffusion weighted MRI}

The quality of DWI images of 51 lesions were reviewed, all had good quality. 12 patients had metastatic renal hilar and para aortic lymph nodes, all showed bright signal intensity in DWI images.

We had 27 malignant lesions out of 28 lesions demonstrated low signal intensity on the DWI images at b 800, only one showed low signal intensity on both DWI and ADC images.

4 lesions showed heterogeneous signal intensity on both DWI and ADC images, due to mixed solid and cystic components. 3 cysts were clear renal cell carcinoma and 1 lesion was unclassified renal cell carcinoma (Figure 3). 
Citation: Saly ZA, Mohamed AEG, Medhat R, Mohamed E (2018) Role of MR Apparent Diffusion Coefficient in Characterization of Cystic Renal Masses Using 3T MRI. J Nucl Med Radiat Ther 9: 383. doi:10.4172/2155-9619.1000383

Page 4 of 5

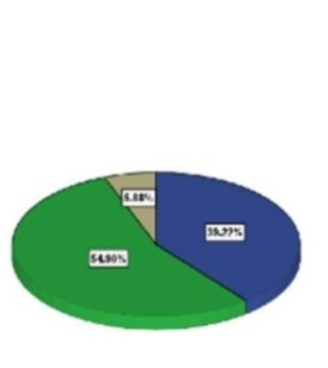

Figure 3: Pie chart for distribution of renal cysts.

\section{ADC values}

The normal renal parenchyma showed mean ADC values was $2.07 \times$ $10^{-3}$ and $1.56 \times 10^{-3} \mathrm{~mm}^{2} / \mathrm{s}$ at b800 and $1600 \mathrm{~s} / \mathrm{mm}^{2}$, respectively.

We found that the benign cystic lesions were significantly high compared to malignant cystic lesions $3.03 \times 10^{-3} \mathrm{~mm}^{2} / \mathrm{s}(0.75)(1.67 \times$ $\left.10^{-3} \mathrm{~mm}^{2} / \mathrm{s}\right)(0.61) 10^{3}$ versus $1.9(0.59) 10^{3} \mathrm{~mm}^{2} / \mathrm{s}(0.59)\left(1.12 \times 10^{-3}\right.$ $\mathrm{mm}^{2} / \mathrm{s}$ ) (0.61) [Median (Min-Max)] respectively (Tables 2,3 and Figure 4).

\begin{tabular}{|l|l|l|l|l|}
\hline & Benign mean (SD) & Malignant mean (SD) & Indeterminate mean (SD) & P value \\
\hline b value $800 \mathrm{~s} / \mathrm{mm}^{2}$ & $1.67(0.36)^{\mathrm{A}}$ & $1.12(0.61)^{\mathrm{AB}}$ & $2.00(0)^{\mathrm{B}}$ & $0.001^{*}$ \\
\hline b value $1600 \mathrm{~s} / \mathrm{mm}^{2}$ & $3.03(0.75)^{\mathrm{A}}$ & $1.9(0.59)^{\mathrm{A}}$ & $2.37(0.15)$ & $<0.001^{*}$ \\
\hline
\end{tabular}

Table 2: Comparison of MRI diffusion in diagnosis of renal cyst. ${ }^{(\mathrm{A}, \mathrm{B})}$ : Indicate statistically significant differences.

\begin{tabular}{|l|l|l|l|l|l|}
\hline & & & & \multicolumn{2}{l|}{ Asymptotic 95\% Confidence Interval } \\
\cline { 5 - 7 } Test Result Variable(s) & AUC & Std. Error & P value & Lower Bound & Upper Bound \\
\hline b value $800 \mathrm{~s} / \mathrm{mm}^{2}$ & 0.824 & 0.071 & 0 & 0.685 & 0.962 \\
\hline b value $1600 \mathrm{~s} / \mathrm{mm}^{2}$ & 0.849 & 0.057 & 0 & 0.738 & 0.961 \\
\hline
\end{tabular}

Table 3: Diagnostic accuracy of MRI diffusion for renal cysts cases.

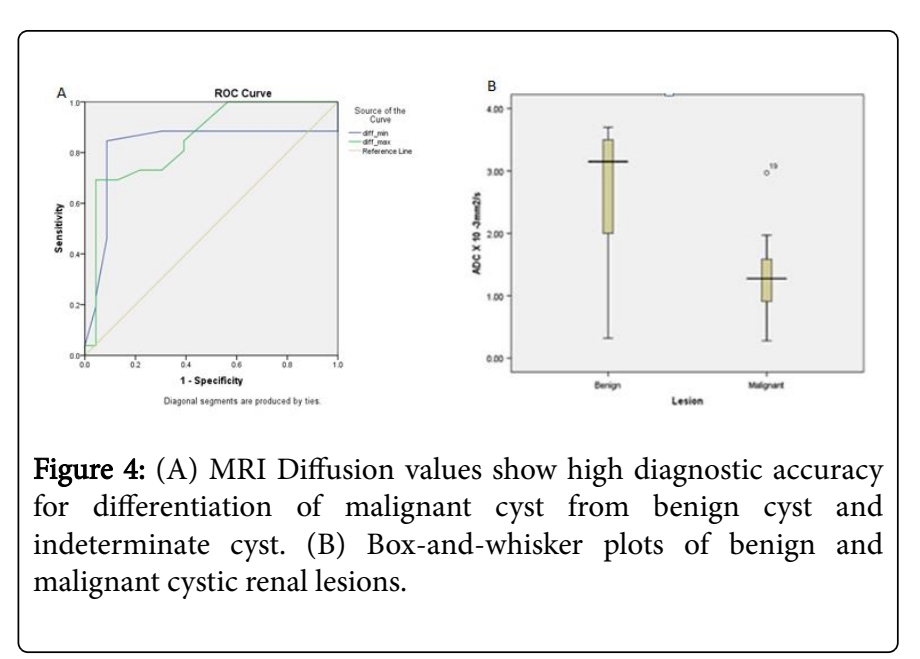

\section{Discussion}

In our study we used DW-MRI trying to characterize the cystic renal masses using ADC values. We found that the ADC values of normal renal parenchyma, benign and malignant renal cysts differ significantly.

Benign cystic renal masses showed high significant values compared to malignant cysts were $3.03 \times 10^{-3} \mathrm{~mm}^{2} / \mathrm{s}\left(0.75 \times 10^{-3} \mathrm{~mm}^{2} / \mathrm{s}\right) 10^{3}$ versus $1.9(0.59) 10^{3} \mathrm{~mm}^{2} / \mathrm{s}$ respectively, and also higher to the normal renal parenchyma which was $2.09 \times 10^{-3}$ at b value $1600 \mathrm{~s} / \mathrm{mm}^{2}$, and these values are lower than the study done by Goya et al.
We assumed that our study have more population data than this limited cases study, and the results are similar to Inci etal with mean ADC value of $3.09 \pm 0.1410^{3} \mathrm{~mm}^{2} / \mathrm{s}$ in benign renal cysts (Bosniak I) and close to study of Zhang et al. who reported $3.269 \pm 0.6110^{3} \mathrm{~mm}^{2} / \mathrm{s}$ for simple renal cysts.

The ADC value of indeterminate cystic lesions at b value $800 \mathrm{~s} / \mathrm{mm}^{2}$ $1600 \mathrm{~s} / \mathrm{mm}^{2}$ are $2.00 \times 10^{-3} \mathrm{~mm}^{2} / \mathrm{s}$ and $2.37 \times 10^{-3} \mathrm{~mm}^{2} / \mathrm{s}\left(0.15 \times 10^{-3}\right.$ $\mathrm{mm}^{2} / \mathrm{s}$ ) respectively, it was lower than the study of Balyemez et al. Who reported The mean ADC value was $2.75 \pm 0.43 \times 10^{-6} \mathrm{~mm}^{2} / \mathrm{s}$ for indeterminate cysts, but this results was limited due to small number of indeterminate cases.

Benign lesions showed significant ADC value difference compared to high malignant renal cysts at $b$ values of $800 \mathrm{~s} / \mathrm{mm}^{2}, 1600 \mathrm{~s} / \mathrm{mm}^{2}$ $(\mathrm{p}<0.001)$, respectively These results are concordant with the study of Goya et al. [12].

Still there is no significant ADC values difference between indeterminate lesions and low malignant lesions (had a low Fuhrman grade $(1$ or 2$)$ at b values of $800 \mathrm{~s} / \mathrm{mm}^{2} 1600 \mathrm{~s} / \mathrm{mm}^{2}(\mathrm{p}=0.001)$ and these results were similar to Balyemez et al.

\section{ROC analysis and cut-off levels}

We found that cut off values for malignant lesions is $1.5 \times 10 \mathrm{~mm} / \mathrm{s}$ ( $\mathrm{p} 0.001$ ) for the ADC with b values of $800 \mathrm{~s} / \mathrm{mm}^{2}$ with sensitivity and specificity $84 \%$ and $92 \%$, respectively and $2.35 \times 10^{-3} \mathrm{~mm}^{2} / \mathrm{s}$ for the ADC with $b$ values of $1600 \mathrm{~s} / \mathrm{mm}^{2}$ ) with sensitivity and specificity of DWI were $73 \%$ and $79 \%$, respectively (Table 4 ). 
Citation: Saly ZA, Mohamed AEG, Medhat R, Mohamed E (2018) Role of MR Apparent Diffusion Coefficient in Characterization of Cystic Renal Masses Using 3T MRI. J Nucl Med Radiat Ther 9: 383. doi:10.4172/2155-9619.1000383

Page 5 of 5

\begin{tabular}{|l|l|l|l|}
\hline Test variable & Cut off & Sensitivity & Specificity \\
\hline b value $800 \mathrm{~s} / \mathrm{mm}^{2}$ & 1.5 & 0.84 & 0.92 \\
\hline b value $1600 \mathrm{~s} / \mathrm{mm}^{2}$ & 2.35 & 0.73 & 0.79 \\
\hline
\end{tabular}

3. Maturen KE, Nghiem HV, Caoili EM, Higgins EG, Wolf JS (2007) Renal mass core biopsy: Accuracy and impact on clinical management. AJR Am J Roentgenol 188: 563-570.

4. Lebret T, Poulain JE, Molinie V, Herve JM, Denoux Y, et al. (2007) Percutaneous core biopsy for renal masses: Indications, accuracy and results. J Urol 178: 1184-1188.

Table 4: Validity of MRI diffusion according to ROC curve.

Bosniak criteria have stood the test of time as Radiologists and Urologists still primarily rely on these guidelines for cystic renal mass evaluation in clinical practice [13]. However recent studies showed that malignancy rates were only $72 \%$ for Bosniak category III lesions and $86 \%$ for Bosniak category IV lesions [14].

DWI MRI in cystic renal masses has been applied successfully for characterization with promising results, still there is limitation for hemorrhagic and proteinaceous cysts with false lower values which may affect both sensitivity and specificity of the ADC measurements. Still post contrast enhanced MRI with subtraction has an important role in this category.

\section{Conclusion}

In conclusion, MRI still have advantages over CT for evaluation of cystic renal masses [15]. The use of 3T MRI is particularly advantageous when parallel imaging is employed (due to higher base line SNR). Increasing fear of post Gadolinium nephrogenic systemic fibrosis makes the radiologist looking for alternative sequences.

DWI-MRI with ADC values as a routine sequence could be useful in characterization of cystic renal masses to avoid unnecessary surgeries as well as a safe sequence for surveillance and follow up. Still more studies are needed for indeterminate renal lesions and benign lesions which are characterized by Bosniak classification as Bosniak III and IV and had unnecessary nephrectomies.

\section{References}

1. Bosniak MA (1986) The current radiological approach to renal cysts. Radiology 158: 1-10.

2. Bosniak MA (1997) The use of the Bosniak classification system for renal cysts and cystic tumors. J Urol 157: 1852-1853.

5. Renshaw AA, Lee KR, Madge R, Granter SR (1997) Accuracy of fine needle aspiration in distinguishing subtypes of renal cell carcinoma. Acta Cytol 41: 987-994.

6. Silverman SG, Gan YU, Mortele KJ, Tuncali K, Cibas ES (2006) Renal masses in the adult patient: the role of percutaneous biopsy. Radiology 240: 6-22.

7. Khan AA, Shergill IS, Quereshi S, Arya M, Vandal MT (2007) Percutaneous needle biopsy for indeterminate renal masses: a national survey of UK consultant urologists. BMC Urol 7: 10.

8. Yang D, Korogi Y, Sugahara T (2002) Cerebral gliomas: Prospective comparison of multivoxel 2D chemical-shift imaging proton MR spectroscopy, echoplanar perfusion, and diffusion weightedMRI. Neuroradiology 44: 656-666.

9. Le Bihan D, Turner R, Douek P, Patronas N (1992) Diffusion MR imaging: Clinical applications. AJR Am J Roentgenol 159: 591-599.

10. Inci E, Hocaoglu E, Aydin S (20121) Diffusion-weighted magnetic resonance imaging in evaluation of primary solid and cystic renal masses using the Bosniak classification. Eur J Radiol 81: 815-820.

11. Zhang J, Tehrani YM, Wang L (2008) Renal masses: Characterization with diffusion-weighted MR imaging-A preliminary experience. Radiology 247: 458-464.

12. Goya C, Hamidi C, Bozkurt Y, Yavuz A, Kudy S (2015) The role of apparent coefficient quantification of differentiating benign and malignant renal masses by $3 \mathrm{~T}$ magnetic resonance imaging. Balkan Med J 32: 273-278.

13. Balyemez F, Aslan A, Inan I, Ayaz E, Karagoz V (2017) Diffusion weighted magnetic resonance imaging in cystic renal masses. Can Urol Assoc J 11: E8-14.

14. Mousessian PN, Yamauchi FI, Mussi TC, Baroni RH (2017) Malignancy rate, histologic grade and progression of Bosniak category III and IV complex renal cystic masses. AJR 209: 1285-1290.

15. Israel GM, Hindman N, Bosniak MA (2004) Evaluation of cystic renal masses: Comparison of CT and MR 417 imaging by using the Bosniak classification system. Radiology 231: 365-371. 